\title{
Spectral and orbital characterisation of the directly imaged giant planet HIP 65426 b`
}

\author{
A. C. Cheetham ${ }^{1}$, M. Samland ${ }^{2}$, S. S. Brems ${ }^{3}$, R. Launhardt ${ }^{2}$, G. Chauvin ${ }^{4,5}$, D. Ségransan ${ }^{1}$, T. Henning ${ }^{2}$, \\ A. Quirrenbach ${ }^{3}$, H. Avenhaus ${ }^{2}$, G. Cugno $^{6}$, J. Girard ${ }^{7}$, N. Godoy ${ }^{8,9}$, G. M. Kennedy ${ }^{10}$, A.-L. Maire ${ }^{2}$, \\ S. Metchev ${ }^{3,11,12}$, A. Müller ${ }^{2}$, A. Musso Barcucci ${ }^{2}$, J. Olofsson ${ }^{8,9}$, F. Pepe ${ }^{1}$, S. P. Quanz ${ }^{6}$, D. Queloz ${ }^{13}$, S. Reffert $^{3}$, \\ E. Rickman ${ }^{1}$, R. van Boekel ${ }^{2}$, A. Boccaletti ${ }^{14}$, M. Bonnefoy ${ }^{4}$, F. Cantalloube ${ }^{2}$, B. Charnay ${ }^{14}$, P. Delorme ${ }^{4}$, \\ M. Janson ${ }^{2,15}$, M. Keppler ${ }^{2}$, A.-M. Lagrange ${ }^{4}$, M. Langlois ${ }^{16,17}$, C. Lazzoni ${ }^{18}$, F. Menard ${ }^{4}$, D. Mesa ${ }^{18,19}$, \\ M. Meyer ${ }^{20}$, T. Schmidt ${ }^{14}$, E. Sissa ${ }^{18}$, S. Udry ${ }^{1}$, and A. Zurlo ${ }^{16,21,22}$
}

(Affiliations can be found after the references)

Received 20 August 2018 / Accepted 18 December 2018

\begin{abstract}
HIP $65426 \mathrm{~b}$ is a recently discovered exoplanet imaged during the course of the SPHERE-SHINE survey. Here we present new $L^{\prime}$ and $M^{\prime}$ observations of the planet from the NACO instrument at the VLT from the NACO-ISPY survey, as well as a new $Y-H$ spectrum and $K$-band photometry from SPHERE-SHINE. Using these data, we confirm the nature of the companion as a warm, dusty planet with a mid-L spectral type. From comparison of its SED with the BT-Settl atmospheric models, we derive a best-fit effective temperature of $T_{\text {eff }}=1618 \pm 7 \mathrm{~K}$, surface gravity $\log g=3.78_{-0.03}^{+0.04}$ and radius $R=1.17 \pm 0.04 R_{\mathrm{J}}$ (statistical uncertainties only). Using the DUSTY and COND isochrones we estimate a mass of $8 \pm 1 M_{\mathrm{J}}$. Combining the astrometric measurements from our new datasets and from the literature, we show the first indications of orbital motion of the companion (2.6 $\sigma$ significance) and derive preliminary orbital constraints. We find a highly inclined orbit $\left(i=107_{-10}^{+13} \mathrm{deg}\right)$ with an orbital period of $800_{-400}^{+1200} \mathrm{yr}$. We also report SPHERE sparse aperture masking observations that investigate the possibility that HIP $65426 \mathrm{~b}$ was scattered onto its current orbit by an additional companion at a smaller orbital separation. From this data we rule out the presence of brown dwarf companions with masses greater than $16 M_{\mathrm{J}}$ at separations larger than $3 \mathrm{AU}$, significantly narrowing the parameter space for such a companion.
\end{abstract}

Key words. stars: individual: HIP 65426 - planets and satellites: atmospheres - techniques: high angular resolution planets and satellites: detection - planetary systems

\section{Introduction}

The number of exoplanets that can be studied through direct imaging is steadily growing (e.g. Macintosh et al. 2015; Chauvin et al. 2017a; Keppler et al. 2018), and each object can provide a wealth of information about their formation, evolution and atmospheres. For studies of giant planet atmospheres, the midinfrared wavelength range covers a critical regime that is particularly sensitive to cloud properties, and contains strong molecular absorption bands from $\mathrm{CH}_{4}$ and $\mathrm{CO}$ (Sharp \& Burrows 2007). Since young giant planets still retain the heat of their formation, this wavelength range also offers favourable flux ratios between planet and host star. This has led to the majority of the directly imaged planets being discovered or studied in this wavelength range, particularly in the $L$-band (e.g. Chauvin et al. 2004; Marois et al. 2008, 2010; Lagrange et al. 2010; Rameau et al. 2013; Skemer et al. 2014; Macintosh et al. 2015; Müller et al. 2018). However, the increasing thermal background at longer wavelengths makes detections beyond the $L$-band much more difficult and only a few planets have published $M$-band photometry, including HR 8799 b,c,d (Galicher et al. 2011), $\beta$ Pic b (Bonnefoy et al. 2013) and 51 Eri b (Rajan et al. 2017).

\footnotetext{
${ }^{\star}$ Based on observations collected at the European Organisation for Astronomical Research in the Southern Hemisphere under ESO programmes 199.C-0065 (PI: Launhardt), 198.C-0209 (PI: Beuzit) and 1100.C-0481 (PI: Beuzit).
}

JWST will be a critical tool for studying such objects at midinfrared wavelengths, with the NIRCAM and MIRI instruments having high-contrast coronagraphic imaging capabilities across 1.8-25 $\mu \mathrm{m}$ (Krist et al. 2007; Boccaletti et al. 2015).

One planet that has yet to be studied in the mid-infrared is HIP 65426 b, recently discovered by the SHINE (SpHere INfrared survey for Exoplanets) survey utilising the SPHERE instrument at the VLT (Chauvin et al. 2017b). Analysis of its $1-2.3 \mu \mathrm{m}$ spectrum shows a likely L5-L7 spectral type, effective temperature of $T_{\text {eff }}=1300-1600 \mathrm{~K}$, low surface gravity and a luminosity consistent with a $6-12 M_{\mathrm{J}}$ planet. Given its placement in the mid-L spectral sequence, it provides an important opportunity to study the atmospheric physics of young giant planets in a regime where complex cloud/dust physics and nonequilibrium chemistry play key roles (e.g. Galicher et al. 2011; Skemer et al. 2014; Currie et al. 2014). This object is also a primary target of a JWST Early Release Science program (Hinkley et al. 2017), which will provide additional photometry in the near- and mid-infrared.

In this paper, we present the results of new mid-infrared imaging taken during the ISPY survey (Imaging Survey for Planets around Young stars; Launhardt et al. 2018, in prep.) with the NACO instrument at the VLT, as well as new near-infrared coronagraphic and Sparse Aperture Masking (SAM) observations obtained with SPHERE. We adopt the stellar parameters of HIP 65426 from Chauvin et al. (2017a). In particular we assume 
Table 1. Observing log.

\begin{tabular}{|c|c|c|c|c|c|c|c|}
\hline UT Date & Instrument & Filter & $\begin{array}{c}\mathrm{NDIT}^{a} \times \mathrm{DIT}^{b} \\
(\mathrm{~s})\end{array}$ & $\begin{array}{c}\operatorname{NDIT}^{a} \times \operatorname{DIT}^{b}\left(\text { Flux }^{c}\right) \\
(\mathrm{s})\end{array}$ & $\begin{array}{c}\Delta \pi^{d} \\
\left({ }^{\circ}\right)\end{array}$ & $\begin{array}{c}\text { True North } \\
\left(^{\circ}\right)\end{array}$ & $\begin{array}{c}\text { Pixel scale } \\
\left(\text { mas pixel }^{-1}\right)\end{array}$ \\
\hline 2017-05-18 & NACO & $L^{\prime}$ & $30114 \times 0.2$ & $2400 \times 0.1$ & 113 & $-0.44 \pm 0.10$ & $27.20 \pm 0.05$ \\
\hline 2017-05-19 & NACO & $M^{\prime}$ & $71200 \times 0.05$ & $2400 \times 0.04$ & 96 & $-0.44 \pm 0.10$ & $27.20 \pm 0.05$ \\
\hline $2017-05-03$ & SPHERE-IFS & $Y-H+\mathrm{SAM}$ & $384 \times 4$ & & 49.5 & $1.78 \pm 0.05$ & $7.46 \pm 0.02$ \\
\hline $2017-05-03$ & SPHERE-IRDIS & $K_{1}+\mathrm{SAM}$ & $720 \times 2$ & & 49.5 & $1.78 \pm 0.05$ & $12.267 \pm 0.01$ \\
\hline $2017-05-03$ & SPHERE-IRDIS & $K_{2}+\mathrm{SAM}$ & $720 \times 2$ & & 49.5 & $1.78 \pm 0.05$ & $12.263 \pm 0.01$ \\
\hline 2018-05-12 & SPHERE-IFS & $Y-H$ & $40 \times 96$ & $47 \times 2$ & 31.7 & $1.76 \pm 0.06$ & $7.46 \pm 0.02$ \\
\hline 2018-05-12 & SPHERE-IRDIS & $K_{1}$ & $40 \times 96$ & $101 \times 0.84$ & 31.7 & $1.76 \pm 0.06$ & $12.267 \pm 0.01$ \\
\hline $2018-05-12$ & SPHERE-IRDIS & $K_{2}$ & $40 \times 96$ & $101 \times 0.84$ & 31.7 & $1.76 \pm 0.06$ & $12.263 \pm 0.01$ \\
\hline
\end{tabular}

Notes. ${ }^{(a)}$ NDIT refers to the total number of integrations. ${ }^{(b)}$ DIT refers to the integration time of each image. ${ }^{(c)}$ Flux refers to the sequence of images taken to measure the number of detector counts and PSF shape of the primary star. ${ }^{(d)} \Delta \pi$ is the maximum change in parallactic angle during the sequence.

a solar metallicity, an effective temperature of $8840 \pm 200 \mathrm{~K}$ estimated from HARPS spectroscopy, as well as a primary mass of $1.96 \pm 0.04 M_{\odot}$ and age of $14 \pm 4 \mathrm{Myr}$ from comparison of isochrones with the photometry of HIP 65426 and that of neighbouring stars. However, we update its parallax and distance using the results of the Gaia DR2 (Gaia Collaboration 2016, 2018). This yields a distance of $109.2 \pm 0.7 \mathrm{pc}$ (c.f. $111.4 \pm 3.8 \mathrm{pc}$ from Chauvin et al. 2017a), and an updated projected separation for the companion of $90 \mathrm{AU}$.

\section{Observations and data analysis}

\subsection{NACO imaging}

HIP 65426 was observed as part of the NACO-ISPY survey (Launhardt et al. 2018, in prep) on 2017-05-18 and 2017-05-19 using the $L^{\prime}(3.8 \mu \mathrm{m})$ and $M^{\prime}(4.8 \mu \mathrm{m})$ filters respectively of the NACO instrument (Lenzen et al. 1998; Rousset et al. 2003). The majority of our observing time consisted of short exposure images with no coronagraph, dithering the star between 3 quadrants of the NACO detector ${ }^{1}$. Despite the short integration time, the stellar PSF was partially saturated on the detector in $L^{\prime}$. More details of the observing sequence can be found in Table 1. We also acquired a separate set of shorter exposure images to measure the stellar flux at the beginning and end of the sequence.

The data were reduced independently using the GRAPHIC (Hagelberg et al. 2016), PynPoint (Amara \& Quanz 2012), and IPAG-ADI (Chauvin et al. 2012) packages. These pipelines follow similar approaches for data cleaning. Similar results were obtained, and so we report the results of the GRAPHIC pipeline. Briefly, first the frames were sky subtracted using principal component analysis (PCA) following the approach of Hunziker et al. (2018). The position of the star was then measured in each frame by fitting a Gaussian profile. We then compared the fitted parameters (amplitude, position and width) across the observing sequence and removed outliers more than 5 median absolute deviations from the median of each parameter. Frames were shifted to be centred on the star using Fourier transforms. The cleaned datacubes were then PSF (point spread function) subtracted using PCA (Soummer et al. 2012; Amara \& Quanz 2012). The cleaning procedure was also performed on the unsaturated,

\footnotetext{
1 We avoided the detector quadrant with persistent striping present since the recommissioning of NACO in 2014-2015. This quadrant is in the lower left, containing pixel $(0,0)$.
}

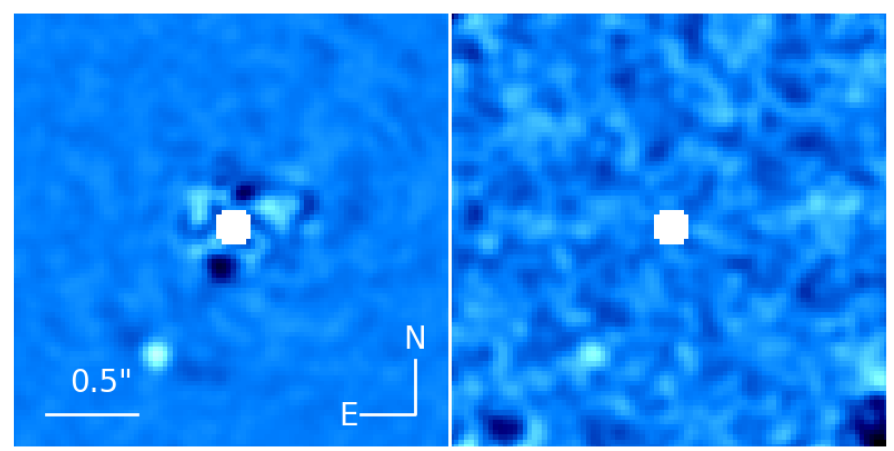

Fig. 1. Final PSF-subtracted images from the two NACO datasets in $L^{\prime}$ (left panel) and $M^{\prime}$ (right panel). The companion is detected in both datasets. While we find a lower contrast ratio in $M^{\prime}$, the high thermal background leads to a lower overall SNR.

shorter exposure images to measure the stellar flux and as a reference for the unsaturated PSF shape.

For the GRAPHIC reduction, the data were first binned in groups of 126 frames for the $L^{\prime}$ data and groups of 200 frames for the $M^{\prime}$ data. PCA was applied to annular sections of the image, each with a width of $2 F W H M$ (full width at half maximum, measured from the stellar PSF). For each frame, a reference library was constructed using only those frames where the field rotation was large enough for a companion to have moved by 0.75 FWHM. $30 \%$ of the available PCA modes were then subtracted from the data. The PSF-subtracted frames were then derotated using Fourier transforms (Larkin et al. 1997) and median-combined to produce the final images in Fig. 1.

To extract the astrometry and photometry of the companion, we used the negative PSF injection technique (Lagrange et al. 2010). For each set of parameters, we inject a negative copy of the unsaturated PSF into the raw frames with the appropriate position and flux. We then calculate the likelihood from the residuals in a small (10 pixel) box around the companion position. We assume Gaussian distributed residuals with a standard deviation measured from the values in an annulus at the same separation taken from the initial reduction, after masking out the companion. The same values were used to verify the Gaussianity with a one-sided Kolmogorov-Smirnov test $(D=0.022, p=0.45$ and $D=0.019, p=0.56$ for $L^{\prime}$ and $M^{\prime}$, respectively). The likelihood was measured on a grid of parameters around the best-fit position, and we extracted the uncertainties from the marginal likelihood distribution of each parameter. 
Table 2. Measured photometry of HIP $65426 \mathrm{~b}$ and derived masses.

\begin{tabular}{cccccc}
\hline \hline Instrument & Filter & $\Delta$ mag & App. mag & Abs. mag & App. flux $\left(\mathrm{Wm}^{-2} \mu \mathrm{m}^{-1}\right)$ \\
\hline SPHERE & $H_{2}$ & $11.14 \pm 0.05$ & $17.94 \pm 0.05$ & $12.75 \pm 0.05$ & $(8.6 \pm 0.4) \times 10^{-17}$ \\
SPHERE & $H_{3}$ & $10.78 \pm 0.06$ & $17.58 \pm 0.06$ & $12.39 \pm 0.06$ & $(10.1 \pm 0.6) \times 10^{-17}$ \\
SPHERE & $K_{1}$ & $10.19 \pm 0.10$ & $17.01 \pm 0.09$ & $11.82 \pm 0.09$ & $(7.5 \pm 0.6) \times 10^{-17}$ \\
SPHERE & $K_{2}$ & $9.82 \pm 0.10$ & $16.79 \pm 0.09$ & $11.60 \pm 0.09$ & $(7.1 \pm 0.6) \times 10^{-17}$ \\
NACO & $L^{\prime}$ & $8.47 \pm 0.14$ & $15.26 \pm 0.15$ & $10.07 \pm 0.15$ & $(4.1 \pm 0.5) \times 10^{-17}$ \\
NACO & $M^{\prime}$ & $8.2 \pm 0.4$ & $15.1 \pm 0.5$ & $9.9 \pm 0.5$ & $(2.1 \pm 0.8) \times 10^{-17}$ \\
\hline
\end{tabular}

In addition to the uncertainties resulting from the fit, we explored the effect of different PCA parameters, varying the protection angle between 0.5-1.5 FWHM and the number of PCA modes from 10 to $40 \%$ of the number of binned frames. The scatter of the best-fit values was added in quadrature to our astrometric and photometric uncertainties. We also added an additional term to the photometric uncertainty $\left(13 \%\right.$ in $L^{\prime}$ and $3 \%$ in $M^{\prime}$ ) due to the change in flux of the primary star measured during the short-exposure sequence at the start and end of the observing sequence, caused by variations in the observing conditions and adaptive optics correction. The larger variation seen in $L^{\prime}$ was caused by the presence of thin clouds during the sequence.

To verify the robustness of the uncertainties calculated from this approach, we injected synthetic companions using the PSF of another star observed during the same night. First, we used the best-fit parameters of HIP $65426 \mathrm{~b}$ to remove the companion from the data and generate a clean datacube. We then injected companions with randomly drawn parameters, using fluxes and separations centred on that of HIP $65426 \mathrm{~b}$, and with random position angles. For each iteration we performed the same procedure to measure the parameters of the injected companion. This was repeated 50 times for each filter. We then calculated the difference between the injected and measured parameters. The root-mean-square of these residuals were within $10 \%$ of our uncertainties for HIP $65426 \mathrm{~b}$, and so we conclude that they are representative of our measurement accuracy.

To convert the contrast measurements of HIP $65426 \mathrm{~b}$ to physical fluxes we produced a synthetic spectrum by scaling a BT-NextGen spectrum (Allard et al. 2012) with an effective temperature $T_{\text {eff }}=8800 \mathrm{~K}$, surface gravity $\log g=4.5$ and solar metallicity to the photometry of HIP 65426 compiled from 2MASS, Tycho-2, HIPPARCOS and WISE (Skrutskie et al. 2006; Høg et al. 2000; van Leeuwen 2007; Wright et al. 2010). The contrast ratio measurements were then multiplied by the predicted flux of the primary star in each filter. We then used the Gaia DR2 distance of 109.2 \pm 0.7 pc (Gaia Collaboration 2016, 2018) to convert to absolute flux, with the results given in Table 2 .

\subsection{SPHERE coronagraphic imaging}

As part of the SHINE exoplanet survey (Chauvin et al. 2017b), HIP 65426 was re-observed on 2018-05-12 with deep coronagraphic imaging to improve the $Y-H$ spectrum and $K$-band photometry of the companion, and to monitor its relative astrometry. We used the infrared dual-band imager and spectrograph (IRDIS; Dohlen et al. 2008) and integral field spectrograph (IFS; Claudi et al. 2008) modules of SPHERE (Beuzit et al. 2008). Data were obtained using the IRDIFS EXT mode. In this configuration, IRDIS and IFS operate simultaneously, with IRDIS in dual-band imaging mode (Vigan et al. 2010) using the $K_{1}$ and $K_{2}$ filters (2.100 and $2.251 \mu \mathrm{m}$, respectively) and IFS covering the $Y-H$ bands $(0.96-1.64 \mu \mathrm{m})$.

The data processing follows the same procedure as in Chauvin et al. (2017a). The data were first cleaned using the SPHERE Data Reduction and Handling (DRH) pipeline (Pavlov et al. 2008), consisting of background subtraction, flat fielding and extraction of the spectral data cube. Additional cleaning steps for IFS were performed using the routines described in Mesa et al. (2015), consisting of bad pixel correction, spectral cross-talk correction and an improved wavelength calibration procedure.

The SpeCal pipeline (Galicher et al. 2018) was used to perform the starlight subtraction and companion analysis. The TLOCI (Marois et al. 2014) algorithm was used to perform the starlight subtraction, and we used the PSF model approach described in Galicher et al. (2018) to measure the companion position and flux in each filter.

\subsection{SPHERE aperture masking}

During the SHINE survey, HIP 65426 was also observed with the sparse aperture mask (SAM) mode of SPHERE (Cheetham et al. 2016) on 2017-05-03, using a 7-hole mask. This mode turns the telescope pupil into an interferometric array, allowing the use of observables such as the closure phase (Jennison 1958) that are robust to optical aberrations. This results in SAM being a useful technique for studying high-contrast structure or companions at small angular separations that would otherwise be inaccessible. While it was expected that HIP 65426 b would not be detectable with SAM due to the large separation and high contrast, the data were obtained to search for additional companions that would be undetectable with other techniques. The observations were taken with the IRDIFS_EXT mode, the same filter combination as the coronagraphic imaging data. Further details of the observing sequence can be found in Table 1 .

To clean the data, a different approach was taken for the IFS and IRDIS modules. The IFS data were cleaned using the same approach as the SPHERE coronagraphic data, while the IRDIS data were cleaned using a custom set of $I D L$ routines consisting of background subtraction, flat fielding and bad pixel correction.

The cleaned datacubes were processed with a similar procedure to that outlined in Tuthill et al. (2000) and Kraus et al. (2008), with modifications made to deal with multi-wavelength data (39 channels for IFS and 2 for IRDIS). Briefly, images were centred and windowed with a super-Gaussian function to limit sensitivity to read-noise. Closure phases were then measured from the Fourier transforms of the images, and calibrated using a weighted sum of those measured on the reference star HD 116664, which was observed alternately with HIP 65426 
Table 3. Measured astrometry of HIP $65426 \mathrm{~b}$.

\begin{tabular}{ccccccc}
\hline \hline UT Date & Instrument & Filter & $\rho(\mathrm{mas})$ & $\rho(\mathrm{AU})$ & $\theta(\mathrm{deg})$ & Reference \\
\hline $2016-05-30$ & SPHERE & $H_{2}$ & $830.4 \pm 4.9$ & $90.7 \pm 0.8$ & $150.28 \pm 0.22$ & Chauvin et al. (2017a) \\
$2016-06-26$ & SPHERE & $H_{2}$ & $830.1 \pm 3.2$ & $90.7 \pm 0.7$ & $150.14 \pm 0.17$ & Chauvin et al. (2017a) \\
$2017-02-07$ & SPHERE & $H_{2}$ & $827.6 \pm 1.5$ & $90.4 \pm 0.6$ & $150.11 \pm 0.15$ & Chauvin et al. (2017a) \\
$2017-02-09$ & SPHERE & $K_{1}$ & $828.8 \pm 1.5$ & $90.5 \pm 0.6$ & $150.05 \pm 0.16$ & Chauvin et al. (2017a) \\
$2017-05-18$ & NACO & $L^{\prime}$ & $832 \pm 3$ & $90.8 \pm 0.7$ & $149.52 \pm 0.19$ & This work \\
$2017-05-19$ & NACO & $M^{\prime}$ & $850 \pm 20$ & $93 \pm 2$ & $148.5 \pm 1.6$ & This work \\
$2018-05-12$ & SPHERE & $K_{1}$ & $822.9 \pm 2.0$ & $89.9 \pm 0.6$ & $149.85 \pm 0.15$ & This work \\
$2018-05-12$ & SPHERE & $K_{2}$ & $826.4 \pm 2.4$ & $90.2 \pm 0.6$ & $149.89 \pm 0.16$ & This work \\
\hline
\end{tabular}

during the same sequence. Correlations between wavelengths (for both IFS and IRDIS) were not considered in this analysis, and so the detection limits are expected to be slightly optimistic.

To determine the limits for companion detection, a Monte-Carlo approach was taken. We generated 10000 simulated closure phase datasets using the measured uncertainties. For each combination of separation, contrast and position angle on a grid of values, we counted the number of non-detections, defined as those simulations where a point source model fit the data better than the corresponding binary model. After marginalising over position angle, we calculated $3.3 \sigma$ detection limits for each separation as the contrast at which $99.9 \%$ of noise simulations result in a non-detection. These were then converted to $5 \sigma$ limits for consistency with the NACO and SPHERE imaging data.

\section{Results}

\subsection{Astrometry}

To calibrate the NACO data, we compared $L^{\prime}$ observations of an astrometric reference field in 47 Tuc taken on 2017-05-19 to the catalog of McLaughlin et al. (2006), resulting in the pixel scale and True North offset reported in Table 1. The final measured astrometry of HIP $65426 \mathrm{~b}$ is given in Table 3 . The measured NACO $L^{\prime}$ separation of $832 \pm 3$ mas corresponds to a projected physical separation of $90.9 \pm 0.7 \mathrm{AU}$ using the updated distance to HIP 65426. For the SPHERE coronagraphic imaging data, we used the astrometric calibration scheme outlined in Maire et al. (2016a), with the pixel scale and True North offset also reported in Table 1 .

Between the SPHERE epochs, we see the first indications of orbital motion, with the observed position changing consistently in the same direction and a maximum of $2.6 \sigma$ deviation between the datapoints. To investigate the orbital parameters consistent with the observed motion, we used the Orbits for the Impatient (OFTI) method of Blunt et al. (2017), based on Bayesian rejection sampling. We applied uniform priors in the epoch of periastron passage $T_{0}$ and argument of periastron $\omega$, a $\sin i$ prior in inclination $i$, and a linearly decreasing prior in the eccentricity $e$ based on the results of Nielsen et al. (2008). To choose the semi-major axis $a$ and position angle of nodes $\Omega$, a single datapoint was chosen randomly and sampled with its given uncertainties, and the orbit scaled and rotated to pass through this point. The period was then calculated assuming a primary mass of $1.96 M_{\odot}$. Each parameter set was accepted or rejected by comparing its likelihood to a number randomly drawn from a uniform probability distribution over the range $(0,1)$. This process was repeated until 10000 orbits had been selected, from which we derived our posterior distributions. As in
Blunt et al. (2017), we increased the fraction of accepted orbits by normalising the likelihood function by the maximum likelihood found from an initial run of the algorithm. We find a $0.5^{\circ}$ offset in position angle between the NACO and SPHERE datasets taken in 2017, much larger than the expected change due to orbital motion and inconsistent with the 2016 and 2018 SPHERE measurements. Similar systematic offsets have been found in other datasets, and may originate from the different choice of astrometric reference fields and star catalogs used for the two instruments. To avoid any problems this may cause, we used only the SPHERE epochs to calculate the likelihood of potential orbits. In addition, we used only the astrometry measured in the IRDIS $H_{2}$ and $K_{1}$ filters, leading to 5 datapoints.

A sample of the resulting orbits are shown in Fig. 2, while the full posterior distributions are shown in Fig. 3. From the posterior distributions, significant constraints can be placed on several of the orbital parameters, and the measured values are given in Table 4. We find an orbital period of $\log (P[d])=5.51_{-0.25}^{+0.37}$ $\left(P=800_{-400}^{+1200} \mathrm{yr}\right)$, implying a semi-major axis of $a=110_{-30}^{+90} \mathrm{AU}$. Orbits close to edge-on are preferred, with a peak at $i=107_{-10}^{+13}$. While the $\sin i$ prior favours highly inclined orbits, the posterior distribution is significantly narrower than the distribution of generated orbits, showing that the inclination measurement is not dominated by the prior. Two families of solutions are found, with consistent parameters except for a $180^{\circ}$ change in both $\Omega$ and $\omega$. This corresponds to our lack of knowledge as to whether HIP 65426 b is moving towards or away from us on its orbit. Due to the short time baseline compared to the orbital period and the lack of curvature in the observed motion, the eccentricity is essentially unconstrained, with the posterior distribution reflecting the linear prior used to draw sample orbits. Since we used a linear prior, the peak occurs at $e=0$ and we instead express the eccentricity as a $1 \sigma$ upper limit $(e<0.43)$. In addition, the orbital eccentricity shows significant correlations with several other parameters, with high eccentricity solutions preferring larger inclinations and shorter periods, for example. A different choice of prior would impact many of the orbital parameters.

\subsection{Atmospheric models}

To estimate the physical parameters of HIP 65426 b we compared the observed spectrum to atmospheric models. We used the Markov chain Monte Carlo procedure described in Samland et al. (2017) to explore the likelihood distribution, interpolating the grid of spectral models at each step.

When comparing to the BT-Settl models (Baraffe et al. 2015), we found best-fit values similar to those of Chauvin et al. $(2017 \mathrm{a})$, with $T_{\text {eff }}=1618 \pm 7 \mathrm{~K}, \log g=3.78_{-0.03}^{+0.04} \mathrm{dex}$ and radius $R=1.17 \pm 0.04 R_{\mathrm{J}}$. This model is compared to the observed SED (spectral energy distribution) of HIP 65426 b in Fig. 4. 

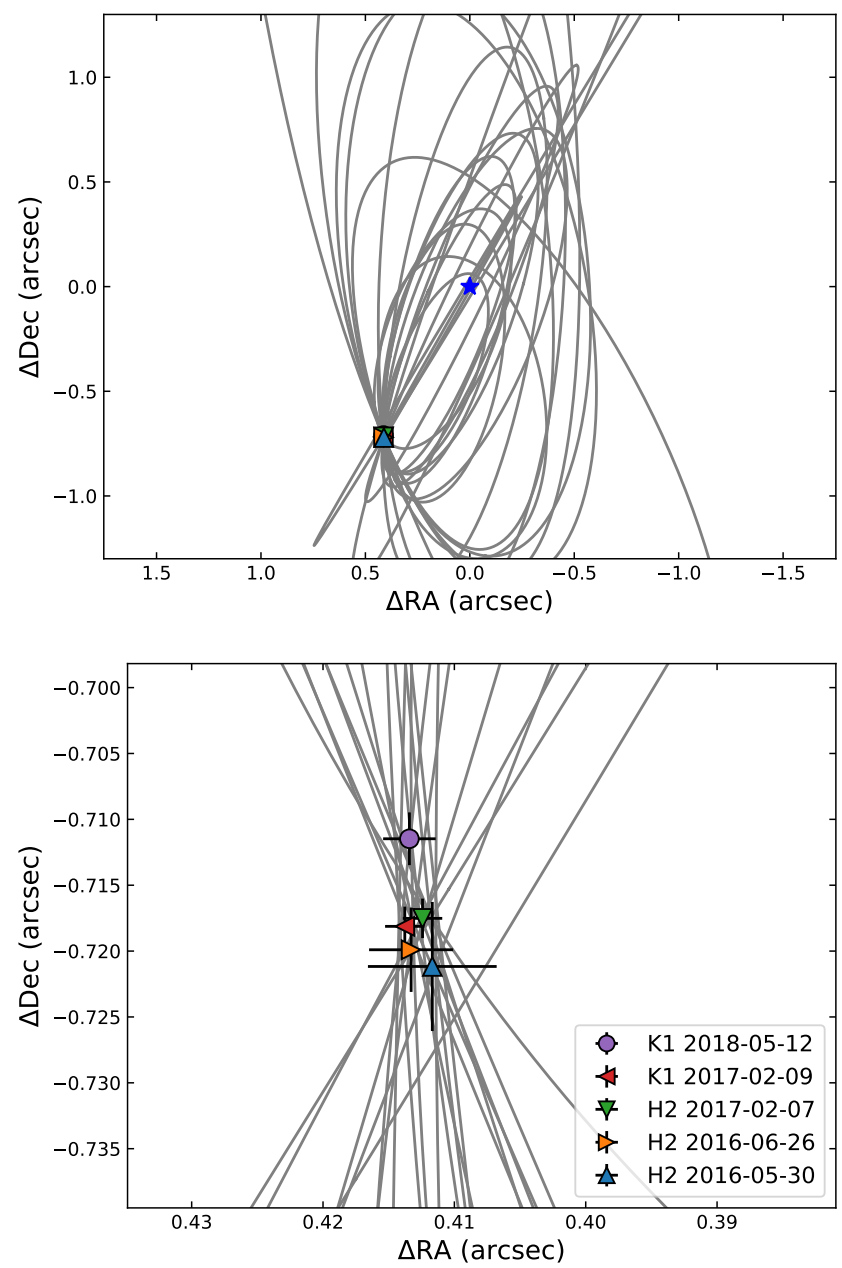

Fig. 2. Observed SPHERE-IRDIS astrometry of HIP 65426 b compared to 20 orbits drawn randomly from the 250 best-fitting orbits in the OFTI output sample. The position of the primary star is shown with a blue star. A close up of the region around the present position of HIP $65426 \mathrm{~b}$ is shown separately in the bottom plot.

Combining the temperature and radius measurements results in a luminosity estimate for HIP 65426 b of $\log \left(L / L_{\odot}\right)=$ $-4.05 \pm 0.03$, while the radius and surface gravity produce a mass esimate of $M=3.32 \pm 0.4 M_{\mathrm{J}}$. The listed uncertainties are optimistic, since they do not account for uncertainties in the modelling of these objects, and different models are likely to show systematic differences in the measured parameters that are larger than the given uncertainties. A number of free parameters are also not included in this analysis, including metallicity and cloud properties, that may impact the results.

We repeated the same procedure as above using the cloudy atmosphere petitCODE models, described in Mollière et al. (2017). We found that the best-fit solution converged to low temperatures $(\sim 1200 \mathrm{~K})$ with unphysically large radii $\left(>2 R_{\mathrm{J}}\right)$, although a second likelihood peak at higher temperatures $(\sim 1800 \mathrm{~K})$ provided a similar fit to the data with different physical parameters. This set of models failed to simultaneously reproduce the shape of the $J$-band peak and the $K$-band flux, and so we concentrate on the BT-Settl results.

\subsection{Empirical comparison}

Using the measured photometry, we placed HIP 65426 b on colour-colour and colour-magnitude diagrams. We compiled spectra of field dwarfs from Leggett et al. (2010), the IRTF spectral library of M, L and T dwarfs (Cushing et al. 2005; Rayner et al. 2009) and the SpeX Prism Library (Burgasser 2014). For targets where no parallax or distance was provided, we used the Database of Ultracool Parallaxes maintained by Trent Dupuy (Dupuy \& Liu 2012; Dupuy \& Kraus 2013) and the results of Dahn et al. (2017), where available. We also included photometry from Dupuy \& Liu (2012). For targets without $L^{\prime}$ magnitudes, we converted WISE W1 $(3.5 \mu \mathrm{m})$ absolute magnitudes to $L^{\prime}(3.8 \mu \mathrm{m})$ absolute magnitudes using a relation calculated from a linear fit to the targets with photometry in both bands. To compare the photometry of HIP $65426 \mathrm{~b}$ with those of other young targets, we included a range of directly imaged companions from the literature (Chauvin et al. 2004; Bonnefoy et al. 2013, 2016; Mawet et al. 2015; Lagrange et al. 2016; Maire et al. 2016b; Mesa et al. 2016; Rajan et al. 2017; Cheetham et al. 2018).

From the colour-magnitude diagrams in Fig. 5, we can see that HIP $65426 \mathrm{~b}$ occupies a similar position to mid-late L-dwarfs. HIP 65426 b is redder and more luminous than the sequence of field dwarfs with similar spectral types. This property is typical for young field dwarfs (Faherty et al. 2012; Liu et al. 2013), which contract and cool over time.

The position of HIP $65426 \mathrm{~b}$ on colour-magnitude diagrams is similar to that of PDS 70 b (Keppler et al. 2018; Müller et al. 2018). At wavelengths longer than $1.4 \mu \mathrm{m}$, their absolute magnitudes are similar. However, HIP 65426 b is significantly brighter in the $J$-band and displays systematically bluer colours than PDS 70 b, suggesting a higher temperature. Given that PDS 70 is also significantly younger than HIP 65426 ( $\sim 5$ and $\sim 14 \mathrm{Myr}$, respectively; Keppler et al. 2018; Chauvin et al. 2017a), the mass of HIP 65426b is expected to be larger.

Compared to other planetary-mass companions, HIP $65426 \mathrm{~b}$ sits clearly between the early L-dwarfs (e.g. $\beta$ Pic b and HD $106906 \mathrm{~b}$ ) and the L/T transition objects (e.g. HR 8799 c,d,e and $2 \mathrm{M} 1207 \mathrm{~b})$. Together, these targets will be useful to study the atmospheric properties of young giant exoplanets across the $\mathrm{L}$ spectral sequence.

\subsection{Mass}

To estimate the mass of HIP $65426 \mathrm{~b}$, we compared the predictions of the DUSTY (Chabrier et al. 2000) and COND (Baraffe et al. 2003) isochrones to the absolute magnitudes presented in Table 2. We used a Monte Carlo procedure to include the uncertainty on the age of the HIP 65426 system, drawing 10000 samples from a Gaussian distribution consistent with the estimated age of $14 \pm 4 \mathrm{Myr}$. We interpolated the isochrones at each age and found the mass that gave the best match to the observed photometry, and took the median and standard deviation of these values as the mass and its uncertainty. With this method, the majority of samples fall below the minimum mass on the DUSTY model grid of $7.3 M_{\mathrm{J}}$, and so we estimate a $1 \sigma$ upper limit of $8.4 M_{\mathrm{J}}$. From the COND models we estimate a mass of $7.5 \pm 0.9 M_{\mathrm{J}}$.

We also used the luminosity estimated from the BT-SETTL model fit of $\log \left(L / L_{\odot}\right)=-4.05 \pm 0.03$, with the estimated age of $14 \pm 4$ Myr. We find values of $8.2 \pm 1.1 M_{\mathrm{J}}$ and $8.3 \pm 0.9 M_{\mathrm{J}}$ for the DUSTY and COND grids respectively.

We find that the colours of HIP $65426 \mathrm{~b}$ are systematically redder than those predicted by the COND isochrones. When placed on a range of colour-magnitude diagrams, such as the one in Fig. 6, the position of HIP 65426 b falls closer to the DUSTY tracks, suggesting the dusty nature of the companion. 


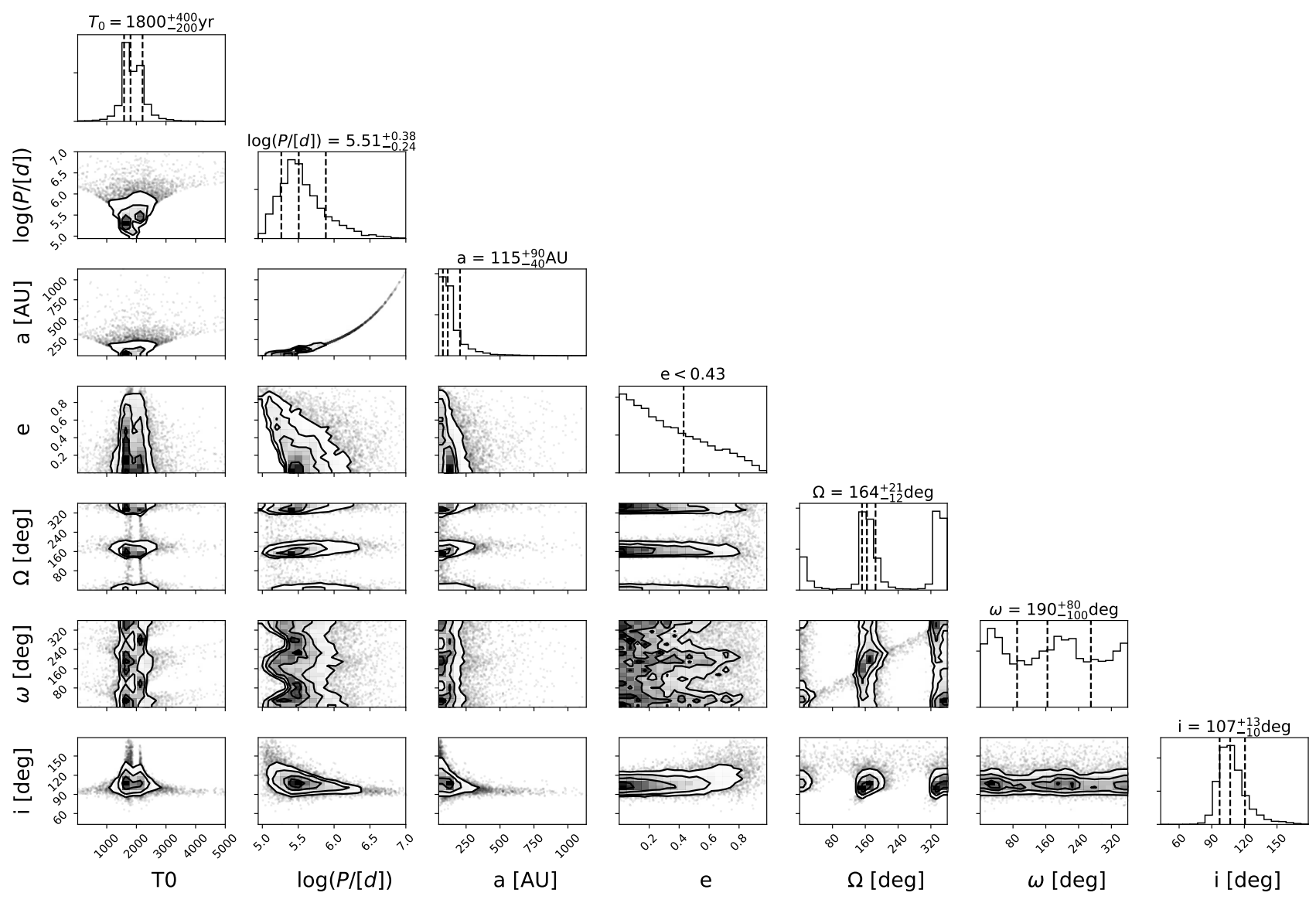

Fig. 3. Posterior distributions for the orbital parameters of HIP $65426 \mathrm{~b}$ around its host star. The dotted lines show the median value and $1 \sigma$ uncertainties for each parameter. For $\Omega$ and $\omega$, the value and uncertainties of one of the two solution families is plotted with a dotted line. For the eccentricity $e$, only the $1 \sigma$ limit is plotted.

Table 4. Measured orbital parameters of HIP $65426 \mathrm{~b}$.

\begin{tabular}{ccc}
\hline \hline Parameter & Solution family 1 & Solution family 2 \\
\hline $\log (P[d])$ & $5.51_{-0.25}^{+0.37}$ & $5.50_{-0.23}^{+0.38}$ \\
$a(\mathrm{AU})$ & $120_{-40}^{+90}$ & $110_{-30}^{+90}$ \\
$T_{0}(\mathrm{yr})$ & $1800_{-200}^{+400}$ & $1800_{-200}^{+400}$ \\
$e$ & $<0.43$ & $<0.43$ \\
$\Omega\left(^{\circ}\right)$ & $164_{-12}^{+21}$ & $344_{-12}^{+20}$ \\
$\omega\left(^{\circ}\right)$ & $190_{-100}^{+80}$ & $20_{-90}^{+80}$ \\
$i\left(^{\circ}\right)$ & $107_{-10}^{+13}$ & $107_{-10}^{+13}$ \\
\hline
\end{tabular}

An in-depth comparison of the photometry of HIP $65426 \mathrm{~b}$ with the BERN exoplanet cooling models by Marleau et al. (2019) resulted in mass estimates of $11.1_{-2.2}^{+1.1} M_{\mathrm{J}}$ and $10.4_{-2.6}^{+0.6} M_{\mathrm{J}}$ for their cold-start and hot-start simulations, suggesting a higher mass than the COND and DUSTY models.

\subsection{Limits on further companions}

We detect no additional companions in the NACO or SPHERE data. The SPHERE SAM detection limits probe a new parameter space not explored by Chauvin et al. (2017a). The $5 \sigma$ detection limits are displayed in Fig. 7. Combining the detection limits with the DUSTY isochrones, we can rule out objects with masses larger than $20 M_{\mathrm{J}}$ at separations of $>2 \mathrm{AU}$, and $16 M_{\mathrm{J}}$ at $>3 \mathrm{AU}$, with a significance of $5 \sigma$.

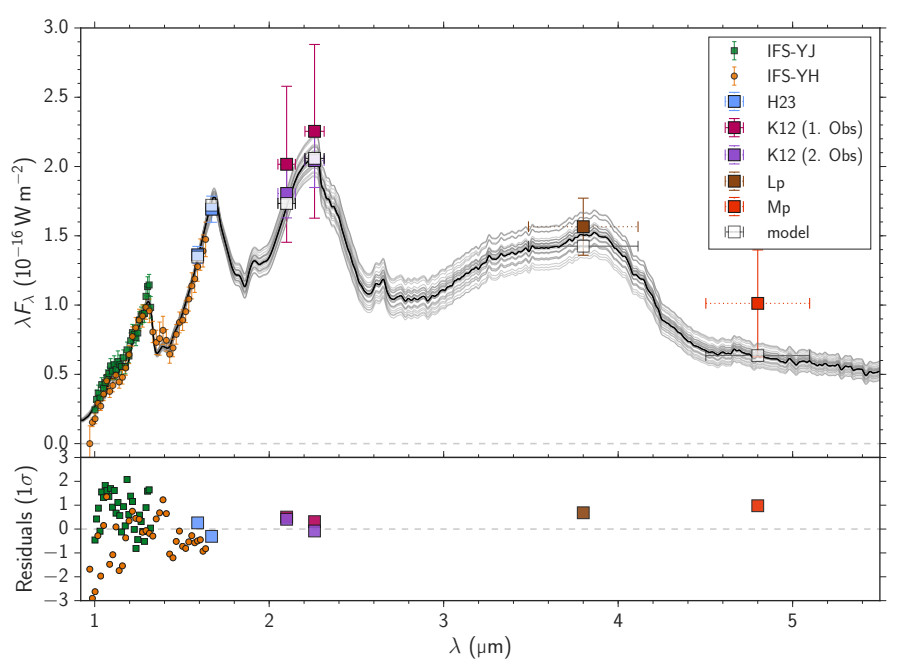

Fig. 4. Observed spectrum of HIP 65426 b (coloured squares) compared to the best-fit spectral model from the BT-Settl grid, with $T_{\text {eff }}=1618 \mathrm{~K}$, $\log g=3.78$ and $R=1.17 \mathrm{R}_{J}$. The horizontal error bars show the width of the SPHERE and NACO filters, and the grey squares show the predicted flux calculated using the filter transmission curves. Several models drawn from the MCMC posterior distributions are also shown in grey.

\section{Summary and conclusions}

In this paper we have presented the first $L^{\prime}$ and $M^{\prime}$ images of the planetary companion HIP 65426 b, extending the coverage 

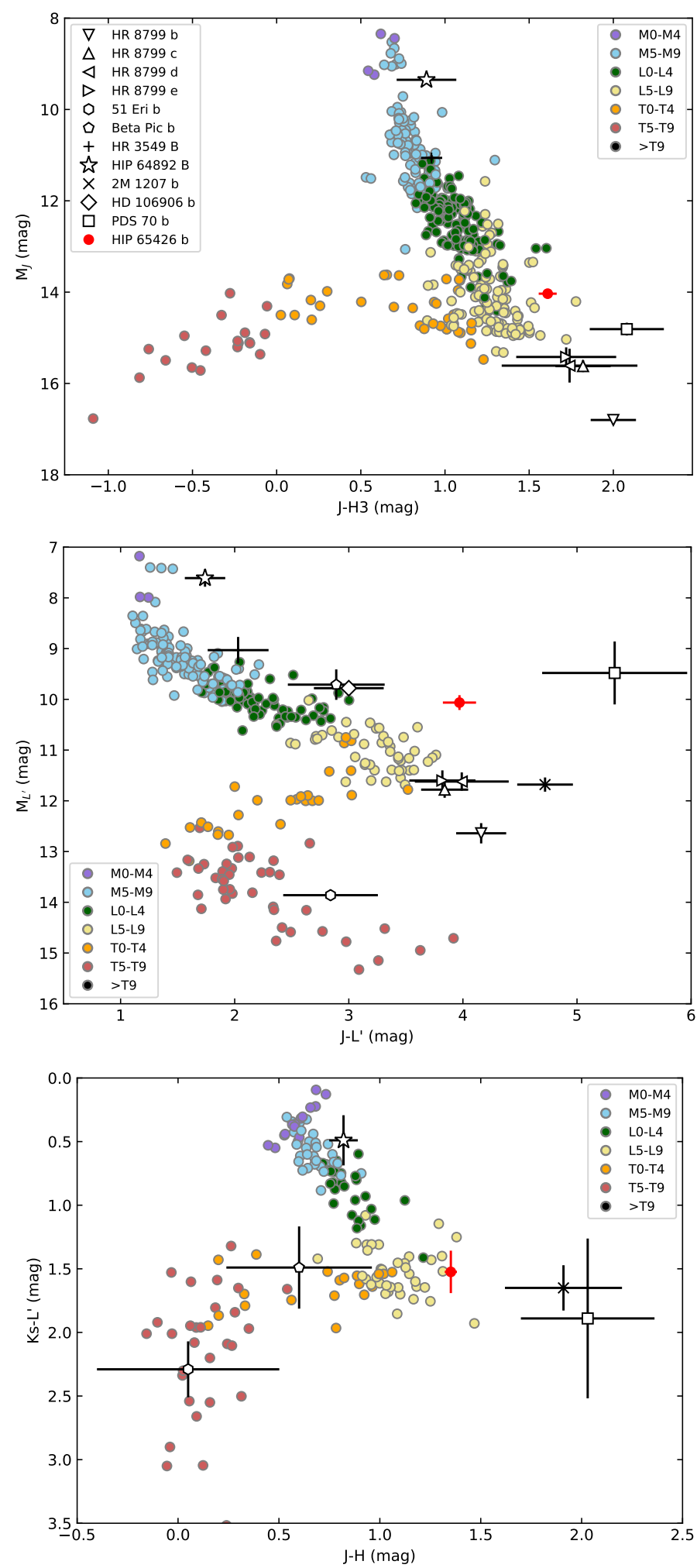

Fig. 5. Colour-magnitude and colour-colour diagrams showing the sequence of field dwarfs (coloured circles), a range of young, imaged brown dwarf and exoplanet companions (black symbols) and HIP 65426 b (red circle). HIP 65426 b falls near objects with mid-L spectral types, but is more luminous and with redder colours than the field sequence. This matches the trend seen for other young objects with similar spectral type.

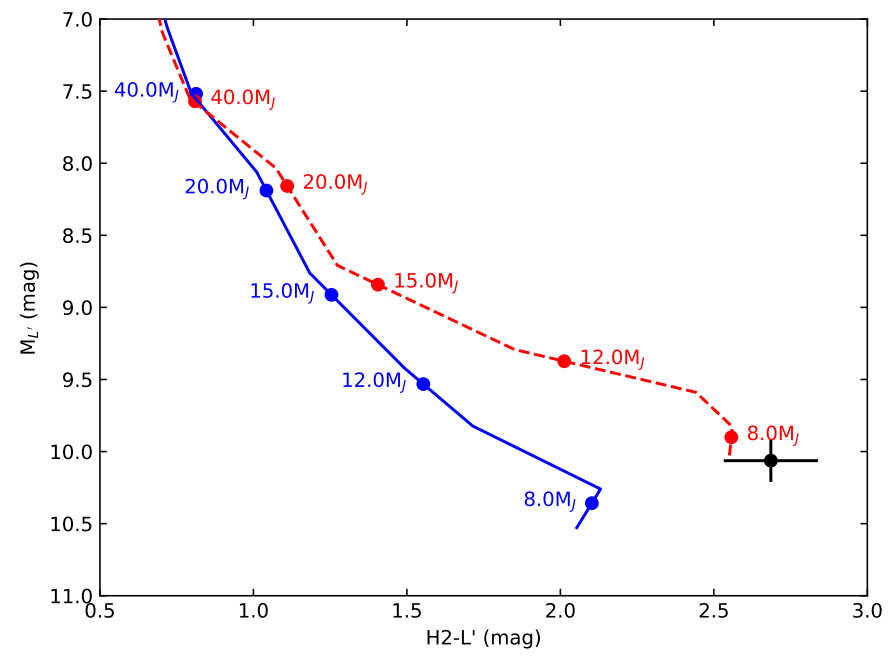

Fig. 6. Colour-magnitude diagram showing the COND (blue) and DUSTY (red) evolutionary models compared to HIP $65426 \mathrm{~b}$. We find that the colours of HIP $65426 \mathrm{~b}$ match the DUSTY models more closely than the COND predictions.
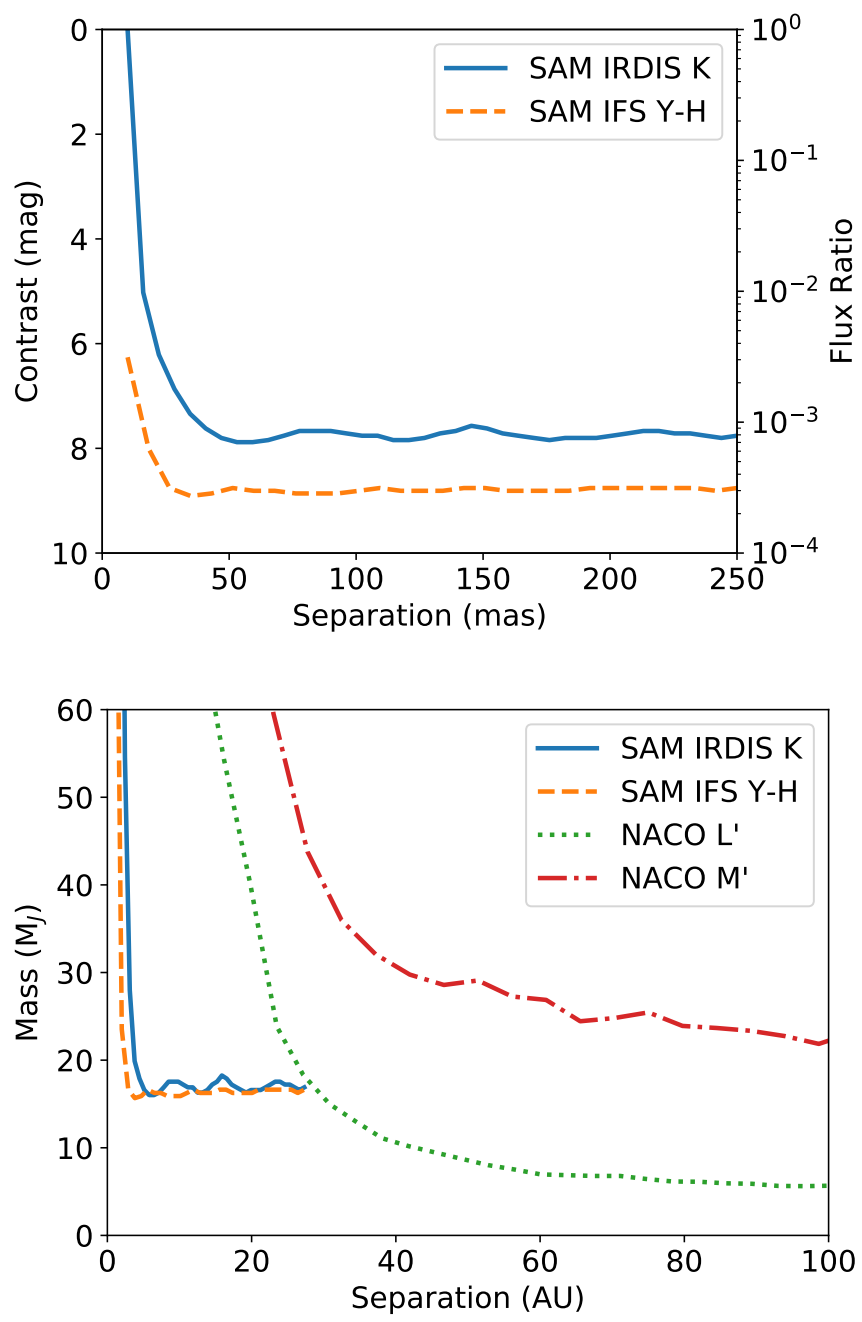

Fig. 7. Detection limits on further companions around HIP 65426. Top panel: detection limits from the SPHERE-SAM data in contrast and angular separation. Bottom panel: mass limits for the SPHERE-SAM and NACO data expressed as projected physical separations. The limits in contrast were converted to masses using the DUSTY isochrones. 
of its SED to the mid-infrared. The photometry extracted from these data are consistent with the conclusions of Chauvin et al. (2017a), confirming the nature of HIP 65426 b as a dusty, warm giant planet with a mid-L spectral type. Through comparison with the BT-Settl models, we find best-fit values of $T_{\text {eff }}=1618 \mathrm{~K}$, surface gravity of $\log g=3.78$ and radius of $R=1.17 R_{\mathrm{J}}$. The observed SED is not fit well by the cloudy petitCODE models, with the grid unable to simultaneously reproduce the features seen at the $J$ and $K$ bands. Using the new thermal-infrared photometry, we update the estimated mass of HIP $65426 \mathrm{~b}$, yielding a value of $8 \pm 1 M_{\mathrm{J}}$ from the DUSTY and COND isochrones. Among these two models, we find that its colours more closely match the predictions of the DUSTY models. This mass estimate is consistent with the results of Marleau et al. (2019) using the Bern exoplanet cooling models.

From the two-year time baseline between datasets, we have obtained the first indications of orbital motion of HIP 65426 b. We performed a preliminary orbital fit, with the present data preferring a close to edge-on orbit $\left(i=107_{-10}^{+13}\right)$ with an orbital period of $P=800_{-400}^{+1200} \mathrm{yr}$. Due to the large orbital period, a significantly longer time baseline will be needed for a full determination of its orbital parameters.

By placing HIP 65426 b on colour-magnitude and colourcolour diagrams, we show that it fits in the sequence of other young giant planets. It displays redder colours and a higher luminosity than the field sequence, a trend typical of young, low gravity, and dusty objects. Amongst the other imaged planets, the recently discovered object PDS $70 \mathrm{~b}$ provides a close match to the observed spectrum of HIP 65426 b and may be a useful analog. These objects join a growing number of planetary-mass objects with L spectral types that have now been imaged. Given that low-surface gravity objects are expected to have cloud layers higher in their atmospheres (Marley et al. 2012), the L-T transition occurs at lower effective temperatures in young planets than field brown dwarfs. In this context, HIP 65426 b is an important object for understanding the atmospheric physics of young giant planets across the L spectral type where clouds are thought to play an important role, and for comparing their properties to that of the better-understood field brown dwarfs.

As discussed in Chauvin et al. (2017a), the large separation of HIP $65426 \mathrm{~b}$ and its high mass are not consistent with in situ formation via core accretion. However, the authors raised the possibility that it may have formed at a much smaller separation and experienced a scattering event with another companion, leading to its present wide orbit. This scenario was explored in detail by Marleau et al. (2019), who found that planet-planet scattering remains a viable pathway to produce objects like HIP 65426 b. Our SPHERE-SAM observations significantly narrow the parameter space for an additional massive object to occupy, ruling out the presence of $>16 M_{\mathrm{J}}$ brown dwarf companions at projected separations of $>3$ AU. However, the current observations cannot discount the scenario of scattering caused by one or more additional giant planets on shorter-period orbits interior to that of HIP $65426 \mathrm{~b}$.

Acknowledgements. This work has been carried out within the frame of the National Centre for Competence in Research "PlanetS" supported by the Swiss National Science Foundation (SNSF). A.M. acknowledges the support of the DFG priority program SPP 1992 "Exploring the Diversity of Extrasolar Planets" (MU 4172/1-1). J.O. and N.G. acknowledge financial support from the ICM (Iniciativa Científica Milenio) via the Núcleo Milenio de Formación Planetaria grant. J.O. acknowledges financial support from the Universidad de Valparaíso, and from Fondecyt (grant 1180395). A.Z. acknowledges support from the CONICYT + PAI/ Convocatoria nacional subvención a la instalación en la academia, convocatoria 2017 + Folio PAI77170087. N.G. acknowledges support from project CONICYT-PFCHA/Doctorado Nacional/2017 folio 21170650.
SPHERE is an instrument designed and built by a consortium consisting of IPAG (Grenoble, France), MPIA (Heidelberg, Germany), LAM (Marseille, France), LESIA (Paris, France), Laboratoire Lagrange (Nice, France), INAF Osservatorio di Padova (Italy), Observatoire Astronomique de l'Université de Genève (Switzerland), ETH Zurich (Switzerland), NOVA (Netherlands), ONERA (France), and ASTRON (Netherlands) in collaboration with ESO. SPHERE was funded by ESO, with additional contributions from CNRS (France), MPIA (Germany), INAF (Italy), FINES (Switzerland), and NOVA (Netherlands). SPHERE also received funding from the European Commission Sixth and Seventh Framework Programmes as part of the Optical Infrared Coordination Network for Astronomy (OPTICON) under grant number RII3-Ct-2004-001566 for FP6 (2004-2008), grant number 226604 for FP7 (2009-2012), and grant number 312430 for FP7 (20132016). This work has made use of the SPHERE Data Centre, jointly operated by OSUG/IPAG (Grenoble), PYTHEAS/LAM/CeSAM (Marseille), OCA/Lagrange (Nice), and Observatoire de Paris/LESIA (Paris) and supported by a grant from Labex OSUG@2020 (Investissements d'avenir ANR10 LABX56). This publication makes use of data products from the Two Micron All Sky Survey, which is a joint project of the University of Massachusetts and the Infrared Processing and Analysis Center/California Institute of Technology, funded by the National Aeronautics and Space Administration and the National Science Foundation. This research has benefitted from the SpeX Prism Spectral Libraries, maintained by Adam Burgasser at http:// pono.ucsd.edu/ adam/browndwarfs/spexprism. This work has made use of data from the European Space Agency (ESA) mission Gaia (https: //www . cosmos.esa.int/gaia), processed by the Gaia Data Processing and Analysis Consortium (DPAC, https://www.cosmos.esa.int/web/gaia/dpac/ consortium). Funding for the DPAC has been provided by national institutions, in particular the institutions participating in the Gaia Multilateral Agreement.

\section{References}

Allard, F., Homeier, D., \& Freytag, B. 2012, Phil. Trans. R. Soc. London, Ser. A, 370, 2765

Amara, A., \& Quanz, S. P. 2012, MNRAS, 427, 948

Baraffe, I., Chabrier, G., Barman, T. S., Allard, F., \& Hauschildt, P. H. 2003, A\&A, 402, 701

Baraffe, I., Homeier, D., Allard, F., \& Chabrier, G. 2015, A\&A, 577, A42 Beuzit, J.-L., Feldt, M., Dohlen, K., et al. 2008, Proc. SPIE, 7014, 701418

Blunt, S., Nielsen, E. L., De Rosa, R. J., et al. 2017, AJ, 153, 229

Boccaletti, A., Lagage, P.-O., Baudoz, P., et al. 2015, PASP, 127, 633

Bonnefoy, M., Boccaletti, A., Lagrange, A.-M., et al. 2013, A\&A, 555, A107

Bonnefoy, M., Zurlo, A., Baudino, J. L., et al. 2016, A\&A, 587, A58

Burgasser, A. J. 2014, ASI Conf. Ser., 11, 7

Chabrier, G., Baraffe, I., Allard, F., \& Hauschildt, P. 2000, ApJ, 542, 464

Chauvin, G., Lagrange, A.-M., Dumas, C., et al. 2004, A\&A, 425, L29

Chauvin, G., Lagrange, A.-M., Beust, H., et al. 2012, A\&A, 542, A41

Chauvin, G., Desidera, S., Lagrange, A.-M., et al. 2017a, A\&A, 605, L9

Chauvin, G., Desidera, S., Lagrange, A.-M., et al. 2017b, in SF2A-2017: Proceedings of the Annual Meeting of the French Society of Astronomy and Astrophysics, eds. C. Reylé, P. Di Matteo, F. Herpin, et al., 331

Cheetham, A. C., Girard, J., Lacour, S., et al. 2016, in Optical and Infrared Interferometry and Imaging V, Proc. SPIE, 9907, 99072T

Cheetham, A., Bonnefoy, M., Desidera, S., et al. 2018, A\&A, 615, A160

Claudi, R. U., Turatto, M., Gratton, R. G., et al. 2008, in Ground-Based and Airborne Instrumentation for Astronomy II, Proc. SPIE, 7014, 70143E

Currie, T., Burrows, A., Girard, J. H., et al. 2014, ApJ, 795, 133

Cushing, M. C., Rayner, J. T., \& Vacca, W. D. 2005, ApJ, 623, 1115

Dahn, C. C., Harris, H. C., Subasavage, J. P., et al. 2017, AJ, 154, 147

Dohlen, K., Langlois, M., Saisse, M., et al. 2008, in Ground-Based and Airborne Instrumentation for Astronomy II, Proc. SPIE, 7014, 70143L

Dupuy, T. J., \& Kraus, A. L. 2013, Science, 341, 1492

Dupuy, T. J., \& Liu, M. C. 2012, ApJS, 201, 19

Faherty, J. K., Burgasser, A. J., Walter, F. M., et al. 2012, ApJ, 752, 56

Gaia Collaboration (Prusti, T., et al.) 2016, A\&A, 595, A1

Gaia Collaboration (Brown, A. G. A., et al.) 2018, A\&A, 616, A1

Galicher, R., Marois, C., Macintosh, B., Barman, T., \& Konopacky, Q. 2011, ApJ, 739, L41

Galicher, R., Boccaletti, A., Mesa, D., et al. 2018, A\&A, 615, A92

Hagelberg, J., Ségransan, D., Udry, S., \& Wildi, F. 2016, MNRAS, 455, 2178

Hinkley, S., Skemer, A., Biller, B., et al. 2017, High Contrast Imaging of Exoplanets and Exoplanetary Systems with JWST, JWST Proposal ID 1386, Cycle 0 Early Release Science

Høg, E., Fabricius, C., Makarov, V. V., et al. 2000, A\&A, 355, L27

Hunziker, S., Quanz, S. P., Amara, A., \& Meyer, M. R. 2018, A\&A, 611, A23

Jennison, R. C. 1958, MNRAS, 118, 276

Keppler, M., Benisty, M., Müller, A., et al. 2018, A\&A, 617, A44 
Kraus, A. L., Ireland, M. J., Martinache, F., \& Lloyd, J. P. 2008, ApJ, 679, 762

Krist, J. E., Beichman, C. A., Trauger, J. T., et al. 2007, in Techniques and Instrumentation for Detection of Exoplanets III, Proc. SPIE, 6693, 66930H

Lagrange, A.-M., Bonnefoy, M., Chauvin, G., et al. 2010, Science, 329, 57

Lagrange, A.-M., Langlois, M., Gratton, R., et al. 2016, A\&A, 586, L8

Larkin, K. G., Oldfield, M. A., \& Klemm, H. 1997, Opt. Commun., 139, 99

Leggett, S. K., Burningham, B., Saumon, D., et al. 2010, ApJ, 710, 1627

Lenzen, R., Hofmann, R., Bizenberger, P., \& Tusche, A. 1998, in Infrared Astronomical Instrumentation, ed. A. M. Fowler, Proc. SPIE, 3354, 606

Liu, M. C., Dupuy, T. J., \& Allers, K. N. 2013, Astron. Nachr., 334, 85

Macintosh, B., Graham, J. R., Barman, T., et al. 2015, Science, 350, 64

Maire, A.-L., Langlois, M., Dohlen, K., et al. 2016a, in Ground-Based and Airborne Instrumentation for Astronomy VI, Proc. SPIE, 9908, 990834

Maire, A.-L., Bonnefoy, M., Ginski, C., et al. 2016b, A\&A, 587, A56

Marleau, G.-D., Coleman, G. A. L., Leleu, A., \& Mordasini, C. 2019, A\&A, in press

Marley, M. S., Saumon, D., Cushing, M., et al. 2012, ApJ, 754, 135

Marois, C., Macintosh, B., Barman, T., et al. 2008, Science, 322, 1348

Marois, C., Zuckerman, B., Konopacky, Q. M., Macintosh, B., \& Barman, T. 2010, Nature, 468, 1080

Marois, C., Correia, C., Véran, J.-P., \& Currie, T. 2014, IAU Symp., 299, 48

Mawet, D., David, T., Bottom, M., et al. 2015, ApJ, 811, 103

McLaughlin, D. E., Anderson, J., Meylan, G., et al. 2006, ApJS, 166, 249

Mesa, D., Gratton, R., Zurlo, A., et al. 2015, A\&A, 576, A121

Mesa, D., Vigan, A., D’Orazi, V., et al. 2016, A\&A, 593, A119

Mollière, P., van Boekel, R., Bouwman, J., et al. 2017, A\&A, 600, A10

Müller, A., Keppler, M., Henning, T., et al. 2018, A\&A, 617, L2

Nielsen, E. L., Close, L. M., Biller, B. A., Masciadri, E., \& Lenzen, R. 2008, ApJ, 674, 466

Pavlov, A., Möller-Nilsson, O., Feldt, M., et al. 2008, in Advanced Software and Control for Astronomy II, Proc. SPIE, 7019, 701939

Rajan, A., Rameau, J., De Rosa, R. J., et al. 2017, AJ, 154, 10

Rameau, J., Chauvin, G., Lagrange, A.-M., et al. 2013, ApJ, 772, L15

Rayner, J. T., Cushing, M. C., \& Vacca, W. D. 2009, ApJS, 185, 289

Rousset, G., Lacombe, F., Puget, P., et al. 2003, in Adaptive Optical System Technologies II, eds. P. L. Wizinowich \& D. Bonaccini, Proc. SPIE, 4839, 140

Samland, M., Mollière, P., Bonnefoy, M., et al. 2017, A\&A, 603, A57

Sharp, C. M., \& Burrows, A. 2007, ApJS, 168, 140

Skemer, A. J., Marley, M. S., Hinz, P. M., et al. 2014, ApJ, 792, 17

Skrutskie, M., Cutri, R., Stiening, R., et al. 2006, AJ, 131, 1163

Soummer, R., Pueyo, L., \& Larkin, J. 2012, ApJ, 755, L28

Tuthill, P. G., Monnier, J. D., Danchi, W. C., Wishnow, E. H., \& Haniff, C. A 2000, PASP, 112,555

van Leeuwen, F. 2007, A\&A, 474, 653

Vigan, A., Moutou, C., Langlois, M., et al. 2010, MNRAS, 407, 71

Wright, E. L., Eisenhardt, P. R. M., Mainzer, A. K., et al. 2010, AJ, 140, 1868

${ }^{1}$ Département d'Astronomie, Université de Genève, 51 chemin des Maillettes, 1290, Versoix, Switzerland e-mail: anthony.cheetham@unige.ch
${ }^{2}$ Max Planck Institute for Astronomy, Königstuhl 17, 69117 Heidelberg, Germany

${ }^{3}$ Landessternwarte, Zentrum für Astronomie der Universität Heidelberg, Königstuhl 12, 69117 Heidelberg, Germany

${ }^{4}$ Université Grenoble Alpes, CNRS, IPAG, 38000 Grenoble, France

5 Unidad Mixta Internacional Franco-Chilena de Astronomía, CNRS/INSU UMI 3386 and Departamento de Astronomía, Universidad de Chile, Casilla 36-D, Santiago, Chile

${ }^{6}$ Institute for Particle Physics and Astrophysics, ETH Zurich, Wolfgang-Pauli-Strasse 27, 8093 Zurich, Switzerland

7 Space Telescope Science Institute, 3700 San Martin Dr. Baltimore, MD 21218, USA

8 Instituto de Física y Astronomía, Facultad de Ciencias, Universidad de Valparaíso, Av. Gran Bretaña 1111, Playa Ancha, Valparaíso, Chile

${ }^{9}$ Núcleo Milenio Formación Planetaria - NPF, Universidad de Valparaíso, Av. Gran Bretaña 1111, Valparaíso, Chile

${ }^{10}$ Department of Physics, University of Warwick, Gibbet Hill Road, Coventry, CV4 7AL, UK

11 Department of Physics and Astronomy, Centre for Planetary Science and Exploration, The University of Western Ontario, London, ON N6A 3K7, Canada

12 Department of Physics and Astronomy, Stony Brook University, Stony Brook, NY 11794-3800, USA

13 Astrophysics Group, Cavendish Laboratory, J.J. Thomson Avenue, Cambridge CB3 0HE, UK

${ }^{14}$ LESIA, Observatoire de Paris, PSL Research University, CNRS, Sorbonne Universités, UPMC Université Paris 06, Université Paris Diderot, Sorbonne Paris Cité, 5 place Jules Janssen, 92195 Meudon, France

15 Department of Astronomy, Stockholm University, AlbaNova University Center, 10691, Stockholm, Sweden

16 Aix-Marseille Université, CNRS, LAM (Laboratoire d'Astrophysique de Marseille) UMR 7326, 13388 Marseille, France

17 CRAL, UMR 5574, CNRS, Université de Lyon, Ecole Normale Supérieure de Lyon, 46 Allée d'Italie, 69364 Lyon Cedex 07, France

18 INAF - Osservatorio Astronomico di Padova, Vicolo dell' Osservatorio 5, 35122, Padova, Italy

${ }^{19}$ INCT, Universidad De Atacama, calle Copayapu 485, Copiapó, Atacama, Chile

20 Department of Astronomy, University of Michigan, 1085 S. University Ave, Ann Arbor, MI 48109-1107, USA

${ }^{21}$ Núcleo de Astronomía, Facultad de Ingeniería y Ciencias, Universidad Diego Portales, Av. Ejercito 441, Santiago, Chile

${ }^{22}$ Escuela de Ingeniería Industrial, Facultad de Ingeniería y Ciencias, Universidad Diego Portales, Av. Ejercito 441, Santiago, Chile 